\title{
BMJ Open Combined cognitive-behavioural and mindfulness programme for people living with dystonia: a proof-of-concept study
}

\author{
H Sandhu, ${ }^{1}$ C J Bernstein, ${ }^{2}$ G Davies, ${ }^{3} \mathrm{~N}$ K Y Tang, ${ }^{4}$ M Belhag, ${ }^{5}$ A Tingle, ${ }^{6}$ \\ M Field, ${ }^{6} \mathrm{~J} \mathrm{Foss}^{7}{ }^{7}$ A Lindahl, ${ }^{5} \mathrm{M}$ Underwood, ${ }^{1}$ D R Ellard ${ }^{1}$
}

To cite: Sandhu $H$,

Bernstein CJ, Davies G, et al. Combined cognitivebehavioural and mindfulness programme for people living with dystonia: a proof-ofconcept study. BMJ Open 2016;6: 011495 .

doi:10.1136/bmjopen-2016011495

- Prepublication history for this paper is available online. To view these files please visit the journal online (http://dx.doi.org/10.1136/ bmjopen-2016-011495).

Received 12 February 2016 Revised 9 May 2016 Accepted 3 June 2016

CrossMark

For numbered affiliations see end of article.

Correspondence to Dr H Sandhu; harbinder.k. sandhu@warwick.ac.uk

\section{ABSTRACT}

Objectives: To design and test the delivery of an intervention targeting the non-motor symptoms of dystonia and pilot key health and well-being questionnaires in this population.

Design: A proof-of-concept study to test the delivery, acceptability, relevance, structure and content for a 3-day group residential programme for the management of dystonia.

Setting: Participants were recruited from a single botulinum toxin clinic. The intervention was delivered in the community.

Participants: 14 participants consented to take part (2 withdrew prior to the starting of intervention). The average age was 60 years (range 44-77), 8 of whom were female. After drop-out, 9 participants completed the 3-day programme.

Intervention: A 3-day group residential programme. Primary and secondary outcome measures:

Process evaluation and interviews were carried out before and after the intervention to explore participant's views and expectations, as well as experiences of the intervention. Select questionnaires were completed at baseline, 1-month and 3-month follow-up.

Results: Although participants were not sure what to expect from the programme, they found it informative and for many this together with being in a group with other people with dystonia legitimised their condition. Mindfulness was accepted and adopted as a coping strategy. This was reflected in the 1-month follow-up.

Conclusions: We successfully delivered a 3-day residential programme to help those living with dystonia manage their condition. Further improvements are suggested. The quantitative outcome measures were acceptable to this group of patients with dystonia.

\section{INTRODUCTION}

Dystonia is one of the most common diagnosed movement disorders after Parkinson's disease, ${ }^{1}$ affecting $\sim 70000$ people in the $\mathrm{UK}^{2}$ It is characterised by involuntary muscle spasm resulting in abnormal

\section{Strengths and limitations of this study}

- We believe this is the first study to test the feasibility of delivering a 3-day residential programme combining a cognitive-behavioural approach and mindfulness to help people living with dystonia.

- The programme was designed in response to a survey by the Dystonia Society which revealed a need for management that addressed the nonmotor symptoms of living with dystonia, such as anxiety and low mood, using a psychological and mindfulness approach.

- The study shows successful recruitment from secondary care.

- However, a limitation is that we only recruited from a single botulinum toxin clinic restricting the population recruited.

- Some participants who were interested could not attend the programme due to the commitment required to the programme.

posturing, usually with a twisting component. ${ }^{3}$ These postures are not fixed and can be associated with slow writhing movements. People with dystonia often use a 'sensory trick' to control the posturing, such as touching the face gently to correct a cervical dystonia. The abnormal posture may be accompanied by tremor. The tremor is often jerky and can vary according to the posture adopted or according to particular tasks. ${ }^{4}$ Task-specific dystonias can be very specific, for example, playing a musical instrument or writing. If people with writer's cramp learn to write with the non-dominant hand, dystonia recurs in about a third of these people. ${ }^{5}$ Dystonia can affect people of all ages. In children, symptoms often start in the limbs and can spread to affect other body parts. Adult-onset ( $>25$ years of age) dystonia usually affects a single part of the body (eg, writer's cramp, torticollis, blepharospasm) or contiguous body parts. ${ }^{3}$ Adult-onset dystonia 
does not tend to be progressive. Primary dystonias are those in which posturing and tremor are the only symptoms and there is no underlying structural or degenerative cause. Secondary dystonia is due to an underlying cause such as brain ischaemia or exposure to certain drugs and heredodegenerative dystonia is where the dystonia forms part of a wider degenerative phenotype. Psychogenic dystonia can be very difficult to distinguish from other forms. ${ }^{4}$

Oral drug treatments for dystonia are often disappointing in terms of efficacy and the emergence of side effects. Trihexyphenidyl, clonazepam, baclofen and tetrabenazine can be tried. Botulinum toxin therapy can be very effective for certain types of dystonia, especially blepharospasm and cervical dystonia. Deep brain stimulation may be considered for patients with idiopathic torsion dystonia and other severe types of dystonia. ${ }^{6}$ All of these treatments, however, focus on the motor symptoms of dystonia.

Non-motor symptoms including mood alterations, cognition (such as attentional functions and memory), sleep impairment and pain ${ }^{7-9}$ are often neglected even though they can account for up to $48 \%$ of the variance in patient outcome (quality of life). Depression in dystonia can be triggered by lack of satisfaction with social support, maladaptive coping strategies, self-depreciation and high levels of disability interfering with everyday activities. ${ }^{10}$ Other psychiatric comorbidities such as anxiety and social phobia have been reported in those with cervical dystonia ${ }^{11}$ (the most commonly diagnosed dystonia), writer's cramp ${ }^{12}{ }^{13}$ and musicians with focal dystonia. ${ }^{14}$ Sleep disturbance is a commonly underdiagnosed problem. ${ }^{15}$ The overall impact of dystonia on quality of life compares to other conditions such as multiple sclerosis, Parkinson's disease, stroke and chronic pain. ${ }^{14}$

Interventions that draw on principles of cognitivebehavioural therapy (CBT) (which address the psychological components mentioned above) can lead to an improvement in the quality of life for various long-term conditions such as chronic fatigue syndrome, chronic pain, diabetes and irritable bowel syndrome ${ }^{16-18}$ and has been shown to be effective for anxiety and mood associated with medical conditions. A survey by the Dystonia Society, $\mathrm{UK}^{2}$ revealed that the majority of its members wanted more information and advice to manage the psychological impact of living with dystonia, including the use of mindfulness (a form of non-judgemental attention to experiences in the present moment) ${ }^{19}$ as a coping strategy. There are however very few data on the role of psychological interventions for dystonia. ${ }^{20}$ Mindfulness practice has been shown to be effective in the management of long-term conditions including chronic pain ${ }^{21}$ and combined with CBT is an effective treatment strategy for conditions with physical and nonphysical symptoms. ${ }^{19} 2223$

We report here the design, development and delivery of a programme combining principles of CBT and mindfulness to a group of adults living with dystonia. We have evaluated the core components of the intervention and overall feasibility of delivering it.

\section{METHODS}

When developing this programme we consulted people living with dystonia recruited through the West Midlands Dystonia Group. Feedback favoured a 3-day residential programme, which would reduce travel to and from the venue for participants and encourage social/friendship bonding within the group. There was also input into the topics chosen for the programme such as understanding and coping with stigma related to dystonia and the impact of dystonia on mood and stress. The programme was delivered over a weekend (Saturday, Sunday and Monday) at a conference venue (University of Warwick), accommodation was provided with breakfast, lunch and dinner for each day of the programme. Feedback favoured a weekend group to allow those with other commitments in the week such as work to be able to attend.

The intervention was designed following guidance from the Medical Research Council (MRC) framework for complex interventions. ${ }^{24}$ This included using theory to inform intervention development and developing a suitable evaluation strategy. In particular, we drew on a cognitive-behavioural approach of exploring thoughts, feelings and behaviours and reframing unhelpful cognitions linked to non-motor symptoms of dystonia and behaviour change. This allowed us to design and map components to specific self-management techniques. This was combined with mindfulness theory and practice. The framework included problem solving, goal setting and coping strategies using self-regulation theory, ${ }^{25}$ the Theory of Planned Behaviour ${ }^{26}{ }^{27}$ and Michie's Behaviour Change Wheel. ${ }^{28}$ The self-regulation theory $^{25}$ draws on an individual's own representation of their illness such as: identity (What is dystonia? What are the symptoms being experienced?), cause (What is the cause of dystonia?), timeline (How long will the dystonia last?), consequences (How will dystonia impact on my life now and in the future?) and control-cure (How effective is the treatment in controlling or curing the dystonia?) which were all addressed within the programme.

Perceived behavioural control (an individual's own belief of ability and skills to carry out a behaviour) is one of the most important and consistent predictors of self-management and behaviour change. ${ }^{29}$ The dystonia intervention incorporated various teaching methods including PowerPoint presentations, group discussions, worksheet and case studies to encourage confidence and increase self-belief. Goal setting was discussed in great detail with participants setting their own goals, which were Specific, Measurable, Achievable, Realistic, Relevant and Time bound (SMART goals). ${ }^{30}$ This was also used as a vehicle to encourage the use of the 
information obtained after the programme had finished. Congruent with Michie's Behaviour Change Wheel ${ }^{28}$ we embedded the intervention in a general framework of education, knowledge and understanding, training of emotional cognitive and behavioural skills, reflective and autonomic motivation and providing an environment for social engagement and support.

The programme was delivered by a multidisciplinary team consisting of psychologists with expertise in management of long-term conditions (HS and NKYT), an expert in mindfulness (GD) and a neurologist who delivered a session explaining dystonia (MB). A participant handbook was produced to supplement the programme. Table 1 outlines the 3-day programme, topics covered and how these were delivered.

\section{Participants}

Patients were recruited from the botulinum toxin clinics, at the University Hospital Coventry and Warwickshire (UHCW); the total recruitment period was 2 months.

\section{Recruitment method}

During the clinic the neurologist briefly screened the patients based on their medical history, and informed them of the study. Those interested were further screened by the researcher (CJB) in a separate room where exclusion and inclusion criteria was applied, consent taken and baseline questionnaires completed.

The inclusion criteria included those with "primary dystonia' for 12 months or longer, those with 'secondary dystonia' were excluded. However, we are aware of the changes with the classification of dystonia according to the Movement Disorder Society (MDS) subsequent to our study being set up. ${ }^{31}$ Participants recruited under the original classification 'primary dystonia' would now map onto the new classification of 'idiopathic adult-onset dystonia'. Participants with a known nervous system pathology or with degenerative causes or with acquired dystonia (axis 2 of the MDS classification) were excluded.

\section{Qualitative/process evaluation}

Face-to-face semistructured interviews were carried out before and after the intervention to explore participants' expectations of the intervention and postintervention, their overall experience. Interviews were carried out by CJB and DRE who were not involved in the facilitation or delivery of the intervention. The interviews were audiotaped and transcribed verbatim; NVivo software was used for managing the analysis. In addition to this, all sessions of the intervention were observed by CJB and DRE, with field notes also contributing to the final analysis.

\section{Measures}

As part of the feasibility, we piloted the use of key questionnaires to collect data in this population:
- Pain and severity of dystonia using the Global Dystonia Rating Scale (GDS) $;^{32}$

- Well-being using the Warwick-Edinburgh Mental Well-Being Scale (WEMWBS) ${ }^{33}$

- Health utilities using the EuroQol EQ-5D-5L; ${ }^{34} 35$

- Mood/psychological distress using the Hospital Anxiety and Depression Scale (HADS) ${ }^{36}$

- Health-related quality of life using the Patient Generated Index (PGI). ${ }^{37} 38$

Each questionnaire was chosen to capture health-related outcomes, in particular we used the GDS, ${ }^{32}$ a reliable and validated scale allowing patients to rate their dystonia severity in 14 body areas. Each body area is rated on a Likert-type scale $(0=$ no dystonia, 1 minimal, 5 moderate and 10 severe). A total score was then calculated (with a maximum of 140). The scale was designed as a simple direct measure assessment of dystonia severity.

The PGI ${ }^{37}$ was used to explore what aspects of their life participants felt were most affected by their dystonia. The description and examples were adapted to make the questionnaire specifically relevant to dystonia with input from lay advisors.

The WEMWBS includes 14 statements rated from 1 (none of the time) to 5 (all of the time) for mental wellbeing which describes positives states of being, including thinking, behaving and feeling. Scoring is calculated by totalling all scores across all items.

The HADS was chosen as a self-report measure of anxiety and depression, and is brief and simple to use. Participants are asked to identify a statement that best describes how they have felt in the last week in relation to various aspects of mood and anxiety.

The scoring of the EQ-5D was no different from its published protocol.

\section{RESULTS}

We invited 28 people living with dystonia to participate in the study, 14 signed up to the actual intervention with 9 completing it. Figure 1 shows the number recruited, withdrawals and final number of participants.

The average age of the participants was 60 years (range 44-77), 8 of whom were female. The group included 10 white Britishers, 1 was Irish and 1 was of mixed race. Five participants were in full-time work, two were in part-time work and five were not working (retired).

The types of dystonia reported by participants varied with all participants reporting dystonia in the neck (cervical dystonia). In addition to this, three of the participants also reported some dystonia in the upper face and eyes, four participants reported dystonia in the jaw and tongue, eight participants reported dystonia in the shoulders and arms, six participants reported dystonia in the larynx and two participants reported dystonia in the feet.

On the first evening of the intervention, one participant left the programme and returned home, and early 
Table 1 Programme structure, content and format of delivery

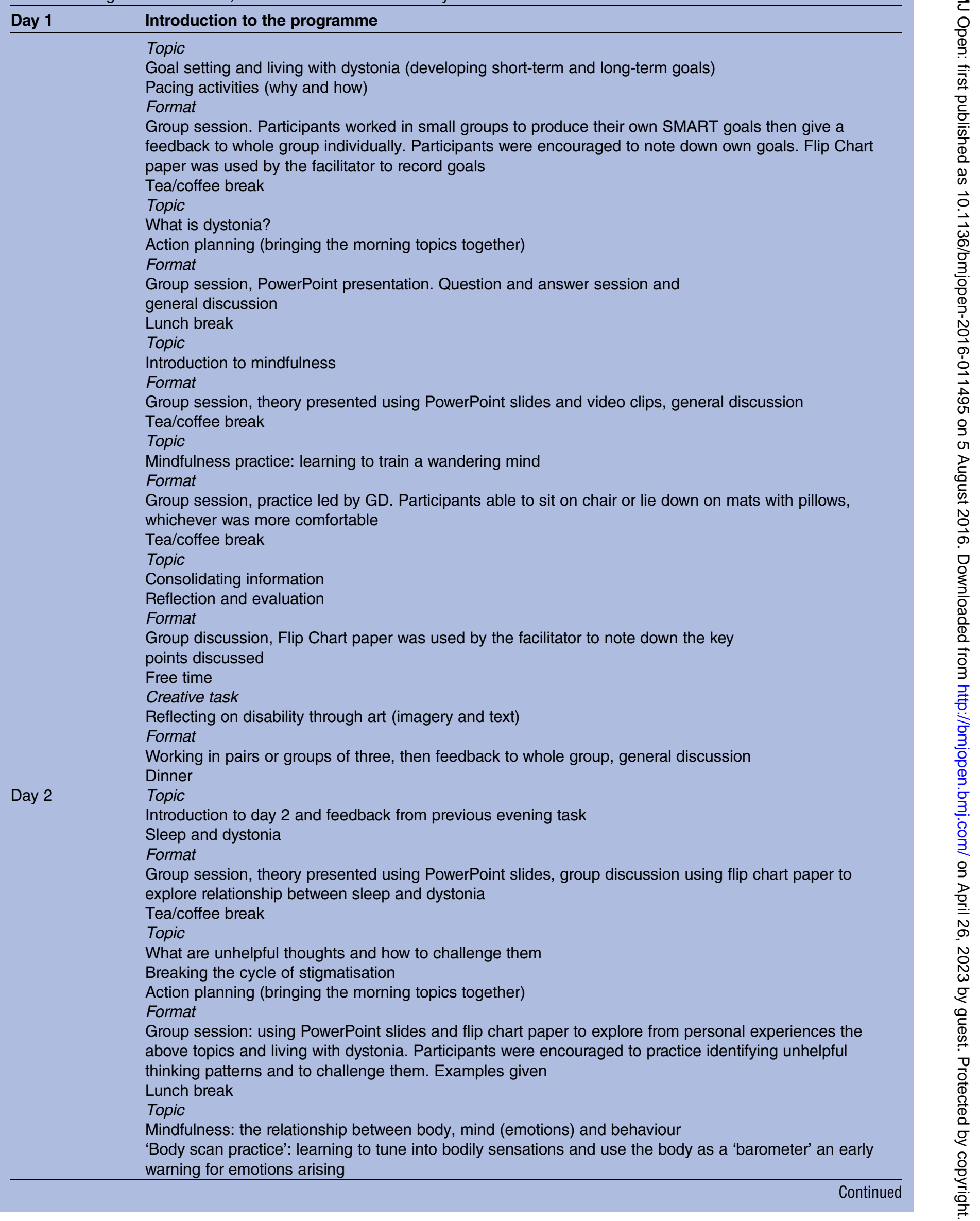


Table 1 Continued

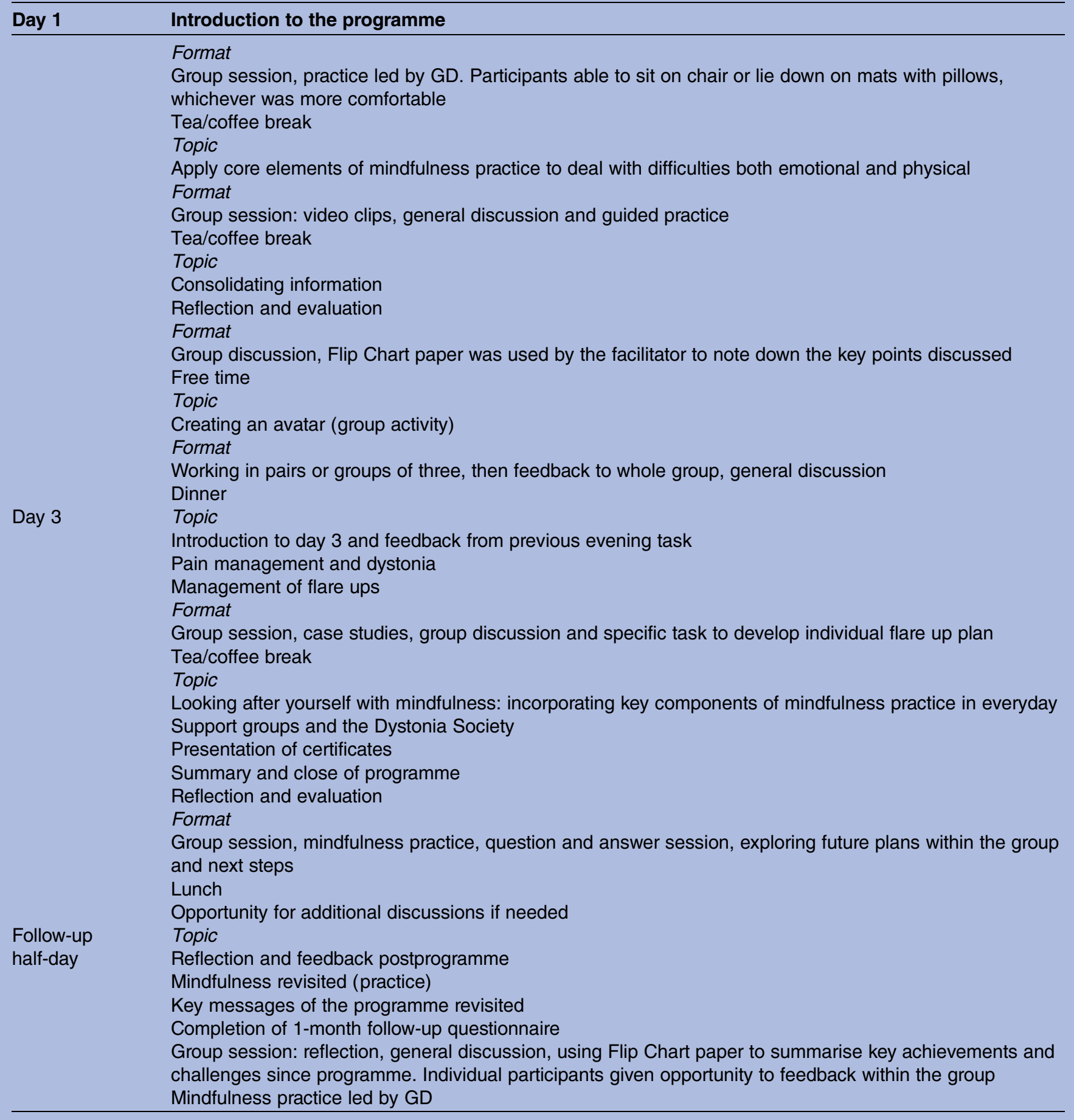

in day 2, a second participant did the same. The team ensured that these participants were safe and well. Both gave very valid reasons for leaving related to their feelings and the severity of their dystonic symptoms. However, we are not presenting these reasons to protect the confidentiality and anonymity of the individuals.

\section{Experiences of living with dystonia}

During the preintervention interviews the participants openly discussed their experiences of living with dystonia from the times before, during and after diagnosis.
They also talked about their relationships with friends, family members and health professionals, and general impact living with dystonia has on their life. This is particularly useful for future adaptations of the programme and further topics were covered as described in table 2.

Briefly below, we outline the experience of the participants that emerged.

\section{Diagnosis experiences}

The participants had lived with dystonia for a number of years ranging from 3 to over 26. From the onset of 


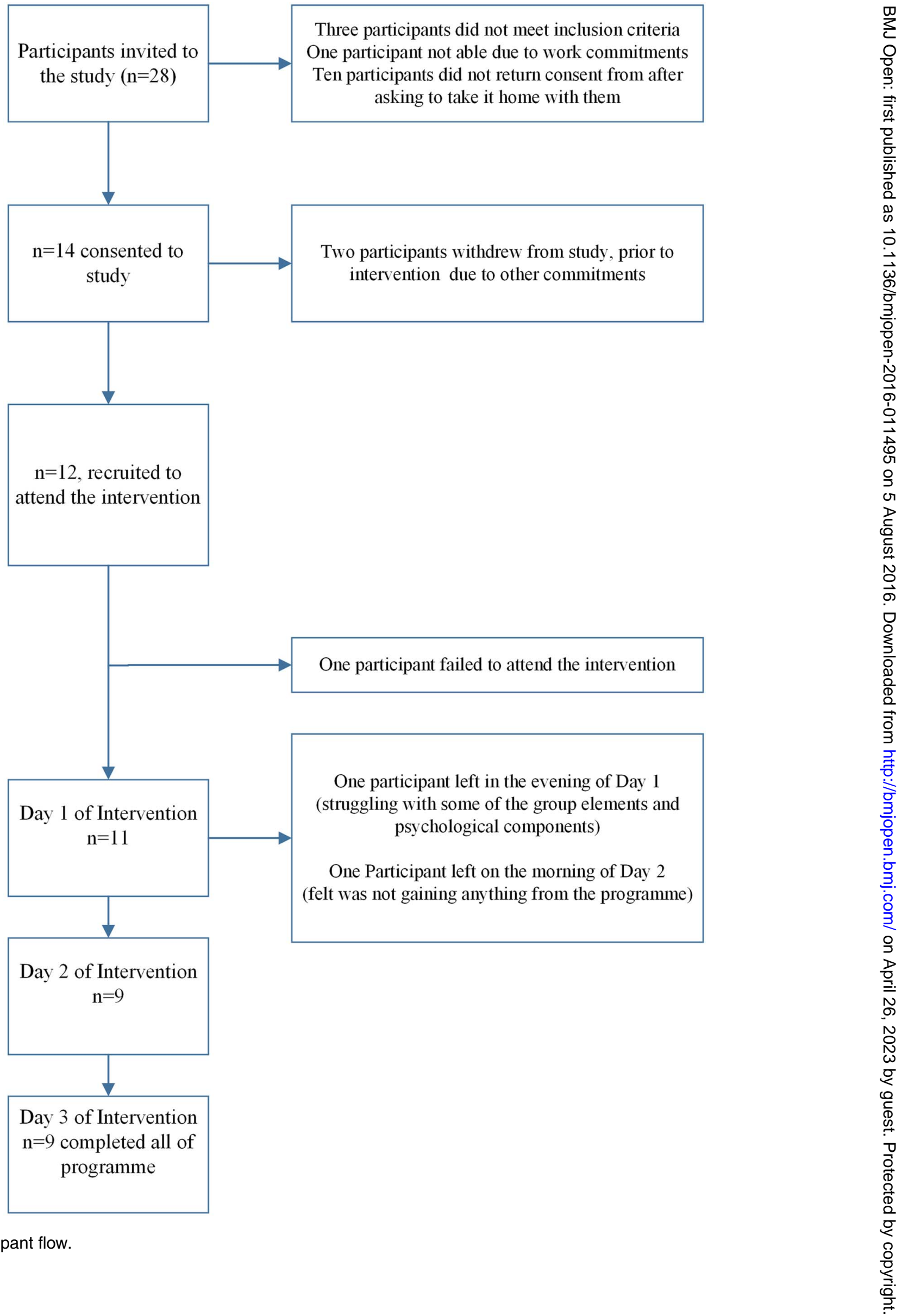

Figure 1 Participant flow. 
Table 2 Summary of key findings and recommendations for future programmes

\section{Summary of key findings from qualitative interviews and lessons leant \\ The variation of delivery mode was positively received during the programme}

Creative tasks-mixed feedback

Understanding and using mindfulness-theory and practice positively received

Group format positively received

\section{Regular breaks needed throughout day}

\section{Positioning of chairs}

Topics were relevant

Preintervention interviews revealed frustration with length of diagnosis

Preinterviews also highlighted the impact of dystonia on day-to-day living

Preintervention interviews revealed acceptance and coping as a particular area which was difficult

\section{Input into future programmes}

To incorporate varied delivery methods of information such as group work to keep participants engaged and allow learning within the group and also individually

Consider the task used for creative learning and group bonding. A task which is unrelated to the topics discussed during the programme To incorporate mindfulness throughout the programme, allowing room for practice and feedback

Small group format, maximum 12 people per group. Allow time for group discussions, facilitation skills important to allow group to learn from each other as well as content of course

To incorporate short regular 5-10 min breaks to be incorporated throughout day giving participants an opportunity to move, stretch Allow participants to position chair within the room or semicircle format, as for some limitations with posture made it difficult to sit and face the front

To incorporate all original topics (plus other outlined below) into the programme which are aimed at improving understanding and self-management of dystonia

To add in topics on communication with healthcare professionals, as this was clearly an area leading to frustration and anxiety and impact on diagnosis

To include topic on problem solving and planning incorporating case studies, scenarios and group work for learning and practice

To incorporate specific elements related to acceptance and education of dystonia as well as coping strategies which could be explored as a group task noticeable symptoms to receiving a formal diagnosis, some reported less than a year being diagnosed with dystonia and others reported over 5 years of repeated visits to clinicians before being diagnosed. Some experienced difficulties in being referred to a specialist because their symptoms were initially labelled as psychosomatic.

My GP had me almost believing that it was (stress), I'd got this tremor because of my home situation, so I found it very disgusting, (my) GP had not particularly taken notice of what I was saying. (P 01)

\section{Impact on life}

All participants reported that receiving a valid diagnosis was a relief although their condition had a far-reaching impact on their lives. Many described living with a number of challenging symptoms such as neck spasms, tremors and pain. Participants also reported experiencing sleep disturbances and poor posture. Consequently, living with dystonia often caused frustration, distress and helplessness.

Just in terms of the psychological because [...] there wasn't anything I could do about it (dystonia) and I was not feeling the same person that I was being portrayed as.... (P 03)

Participants also described feeling unable to fully participate in social activities. In addition, some participants experienced feeling embarrassed as a result of having to manage the visible effects of dystonia in public.

It (dystonia) makes me embarrassed at different stages. You know when you meet new people [...] I've had somebody one time say "you're like a nodding dog"(later) different things that people say and that's very upsetting and then the way people look at you as well. (P 10)

\section{Acceptance and coping}

While the difficulties of living with dystonia were evident, all participants spoke fervently about the importance of managing their condition to overcome some of its challenges. Consequently, acceptance was a significant theme running throughout all participants' accounts. Participants reported using various coping strategies, some participants had found other ways to manage symptoms such as touching their face in order to reduce their symptoms.

What I did find was, I still find it rather strange, if I touch my face that stops it (dystonia).... (P 14)

\section{Expectations of the intervention}

Prior to the intervention, all participants initially reported that they were unsure about what their participation would involve but most said that they hoped the intervention would provide them with more information on dystonia. Others believed that by participating in this 
research they would help reduce the paucity of knowledge currently surrounding dystonia.

Well the reason I'm doing it (participating in the study) [...] (is) because I think it (dystonia) needs more research. Like you see how they research cancer and diabetes but I've never heard of a lot of research into dystonia. (P 12)

While many participants felt that the intervention would be an overall positive experience, some raised concerns:

To be quite honest I'm quite scared to see how bad it (dystonia) can go. (P 10)

The following themes reflect observations during the intervention and postintervention interviews to gain an understanding of experiences and feedback of the intervention.

\section{Delivery of the intervention}

The delivery of the intervention went well overall, with clear presentations and opportunities given to participants to interact with the group. There was a mixture of PowerPoint presentations, group exercises and group discussions which was positively received by participants. Each participant was presented with a completion certificate at the end of the programme and given information about the Dystonia Society.

\section{Creative tasks}

During the 3-day programme, two creative group activities were included as evening tasks. This included interpretation of art (drawings used in literature) and an opportunity to draw an avatar to represent what dystonia meant to them. The participants worked in groups of three and four. There has been suggestion that there is reference to dystonia, for example, in David Copperfield, Dickens depicts characters with generalised dystonia. ${ }^{39}$ However, there was mixed views of the use of such a task in the 3-day programme, with some seeing the relevance and creative element of the task with others feeling it was a little overwhelming.

\section{One-month follow-up session}

One month after the delivery of the intervention, all nine participants who had completed the intervention were invited to a half-day refresher session at the University of Warwick. All participants attended this and generally found it very useful with many are still using the strategies learnt during the 3-day programme including goal setting, mindfulness and challenging unhelpful thinking.

\section{Positive feedback}

For many participants, they felt that the mindfulness sessions were well delivered, informative and beneficial.
Sometimes as I said I might—something has happened it brings all my boys (name) and (name) back (memories of her sons who have died). And I did find I could calm myself a lot more doing these breathing techniques. (P 12)

Supporting the observational data, many participants said that they enjoyed meeting the other people within the group and sharing experiences. Furthermore, one participant noted that they enjoyed the integration of the team with the participants.

The other good thing as well is like (the team), you mixed and stayed with us all the while, as opposed to delivering a lecture and then sort of sitting on a side through the breaks and then come back, and I think the integration there was very good. (P14)

A number of participants noted that they felt that the programme legitimised their illness.

Just really valuable for me to hear that it (dystonia) is a proper thing. It's not just in my head. (P 03)

\section{Improvements and future suggestions}

The team was given suggestions for future programmes including the duration of the programme. Recommendations ranged from making sessions shorter, spreading out the content over the whole 3 days or adopting a different format (eg, weekly sessions over a number of weeks). One participant quoted a 6-week model like the expert patient programme.

This is difficult because you only had the weekend so you (the team) had to cram it in. It's too much. It's too long to be sitting there for that length of time. (Later) I think you need- yes, it needs to be [...] longer [...] more days, yes. (P 06)

The group format was particularly successful with the group gelling very well over the 3 days and planning to continue to meet after the programme and develop their own support network.

Table 2 outlines a summary of key findings of lessons learnt and input into future programme.

\section{Questionnaires}

All 12 participants who originally signed up for the intervention completed a baseline questionnaire. During the 1-month follow-up session, nine participants who completed the programme were provided with a questionnaire and all were returned to the researcher at the end of the session $(100 \%)$. Finally, the nine participants were posted a 3-month questionnaire and seven participants $(78 \%)$ returned the questionnaires.

\section{Pain and severity of dystonia}

Total dystonia severity score measured by GDS showed an improvement from baseline to 3-month follow-up. Each body area is rated on a scale $0-10$, the higher the 
score given the higher the severity of dystonia. Total mean score at baseline was 29.5 (range 7-91), at 1-month follow-up it was 19.3 (range 0-54) and at 3-month follow-up it was 16.6 (range 0-59).

\section{Well-being, mood/psychological distress and anxiety}

Mean scores and SDs for mental well-being (measured by the WEMWBS) and mood and anxiety (measured by the HADS) are presented in table 3, for baseline, 1-month and 3-month follow-up.

\section{Health-related quality of life using the PGI}

Participants were asked at baseline and follow-up to consider five areas of their life that they felt were affected by their dystonia. The most common areas listed were: pain $(n=5)$, sleep $(n=4)$, work $(n=3)$, driving $(n=3)$, hobbies $(n=3)$, housework $(n=2)$, eating and/or drinking $(n=2)$, walking $(n=2)$, relations/relationships $(n=2)$, confidence $(n=2)$ and depression $(n=2)$. Other areas affecting the participants' lives were: movement, relaxation, personal care, standing up straight, looking straight ahead and sexual relationships.

\section{DISCUSSION}

We believe that this is the first study which shows the successful delivery of a combined psychological and mindfulness-based programme to help those living with dystonia. Results from the qualitative analysis and process evaluation indicate that some participants were anxious before the start, not knowing what to expect or even how the programme could help them. However, feedback after the programme showed that there was a greater understanding of what dystonia was and a positive attitude towards the self-management of dystonia and use of mindfulness practice. People with cervical dystonia have reported feelings of self-consciousness, feeling odd or different, unattractive and perceptions of being less confident ${ }^{40}$ challenging unhelpful thinking and stigma was one of the topics within the programme.

We have also successfully demonstrated the acceptability of our main outcome measures to this group. The questionnaires proved useful, in particular the GDS was simple, helped identify the areas of dystonia and severity, and generated an overall score. The PGI identified relevant areas of life effected by dystonia and which ones were perceived as most important such as pain, sleep, work, driving and hobbies. However, there were some missing data which emphasise the need to be able to explain the questionnaire to participants in future studies. It is not possible to draw any inference from our quantitative data. It is, nevertheless, reassuring that the direction of change did not suggest harm, on our selected outcomes. We had a number of participants who left the study during the weekend. This is not unusual or indeed unexpected in a programme like this. A number of participants were very worried about what the weekend would hold for them when we interviewed them before the intervention. It is important within a group setting that participants feel that they have the opportunity to leave should they wish too and that facilitators encourage a relaxed and safe environment for the group. If a participant does leave the group, it is also important that facilitators acknowledge this within the group and then carry on with the rest of the programme. In the current study, some members of the group were worried about the members who left, so addressing this and acknowledging this is important for other members to be able to carry on with the rest of the programme.

In line with the previous literature, this study shows the benefits of targeting specific non-motor symptoms of dystonia, informing and empowering the participants using CBT and mindfulness components. Acceptance was a crucial part of the programme, with those accepting the management of dystonia for long-term benefits engaging more in the programme and completing the whole 3-day programme. The group element was also a success with participants forming a mutual bond over the 3 days and supporting each other throughout the programme including sharing of experiences with the motivation to continue to meet regularly after the programme had finished.

\section{Strengths and weaknesses}

The strengths of the study show the successful recruitment and implications of a 3-day residential programme for people living with dystonia. There were no major incidents during the 3 days and a facilitator was always on call. Limitations of the study include the recruitment method, for this feasibility study we recruited only from one botulinum toxin clinic, restricting our population sample, and most participants who consented to take part had some level of dependent living. For this proof-of-concept study, we only required a small number of participants; we expected that there would be a

Table 3 Mean scores for mental well-being, mood and anxiety

\begin{tabular}{llcc}
\hline & $\begin{array}{l}\text { Mean (SD) } \\
\text { Baseline }\end{array}$ & $\begin{array}{l}\text { Mean (SD) } \\
\text { 1 month }\end{array}$ & $\begin{array}{l}\text { Mean (SD) } \\
\mathbf{3} \text { months }\end{array}$ \\
\hline Well-being score (as measured by WEMWBS) & $44.7(21.65)$ & $47.67(19.46)$ & $51.57(12.91)$ \\
Anxiety score (as measured by HADS) & $8.33(6.05)$ & $5.08(5.53)$ & $4.25(6.03)$ \\
Depression score (as measured by HADS) & $3.92(3.15)$ & $3.25(3.47)$ & $2.42(3.37)$ \\
\hline HADS, Hospital Anxiety and Depression Scale; WEMWBS, Warwick-Edinburgh Mental Well-Being Scale.
\end{tabular}


maximum of 12 people in a group. Drop-out was also a limitation; however, it is expected that we would have some drop-out on such programmes. The interviews with participants demonstrated to us that living with dystonia impacts on many aspects of the person's life and our growing understanding of this will inform our research in the future. In a future study, we would want to widen our recruitment to those attending dystonia support groups.

\section{Implications}

The study has implications for an integrated model of care for those living with dystonia. The qualitative interviews have highlighted diagnosis and referral to specialist care as a huge barrier and concern for those who were recruited. The next stage in this research is a randomised controlled trial to test the effectiveness and costeffectiveness of intervention and patient outcomes, taking on board the lessons learnt and feedback from this study.

\section{CONCLUSION}

Overall, the results of the study encourage further research into an integrated model of care, specifically targeting non-motor symptoms of dystonia. A selfmanagement programme incorporating CBT and mindfulness went above and beyond the expectations of the participants recruited. The feedback is useful for future adaptions to the programme.

\author{
Author affiliations \\ ${ }^{1}$ Clinical Trials Unit, Warwick Medical School, University of Warwick, Coventry, \\ UK \\ ${ }^{2}$ Division of Health Sciences, Warwick Medical School, University of Warwick, \\ Coventry, UK \\ ${ }^{3}$ University of Southampton, Southampton, UK \\ ${ }^{4}$ Department of Psychology, University of Warwick, Coventry, UK \\ ${ }^{5}$ Department of Neurology, University Hospitals Coventry and Warwickshire \\ NHS Trust, Coventry, UK \\ ${ }^{6}$ The Dystonia Society (West Midlands Group), West Midlands, UK \\ ${ }^{7}$ Department of Computer Science, University of Warwick, Coventry, UK
}

Twitter Follow Harbinder Sandhu at @DrHSandhu

Acknowledgements The authors would like to thank the Dystonia Society for funding this study and their ongoing support with the project. They would also like to thank the West Midlands Dystonia Group for their valuable input and support and in particular the lay members who formed part of the study team. They would also like to thank the Development and Alumni Relations Office (DARO) for their support with the project. This project benefited from facilities funded through Birmingham Science City Translational Medicine Clinical Research and Infrastructure Trials Platform, with support from Advantage West Midlands.

Contributors All authors have contributed to the design of the study and manuscript preparation. HS, DRE and CJB were also involved in the analysis and interpretation of data. HS was principal investigator and led the project. HS, NKYT and GD contributed to the design and delivery of the intervention. $\mathrm{JF}, \mathrm{AT}$ and MF were lay advisors and contributed to the intervention design and manuscript preparation. MB and AL were involved in the recruitment, MB was involved in the delivery of the intervention and writing of the manuscript. MU contributed to study design, running of project and writing of the manuscript.
Funding The study was funded by the Dystonia Society (registered charity 1062595).

Competing interests All authors have completed the ICMJE uniform disclosure form at http://www.icmje.org/coi_disclosure.pdf and declare: financial support from the Dystonia Society for the study (DRE and CJB).

Ethics approval The National Health Service (NHS) ethical approval was given by the National Research Ethics Service (NRES) Committee East MidlandsLeicester, REC Number: 14/EM/1091.

Provenance and peer review Not commissioned; externally peer reviewed.

Data sharing statement No additional data are available.

Open Access This is an Open Access article distributed in accordance with the Creative Commons Attribution Non Commercial (CC BY-NC 4.0) license, which permits others to distribute, remix, adapt, build upon this work noncommercially, and license their derivative works on different terms, provided the original work is properly cited and the use is non-commercial. See: http:// creativecommons.org/licenses/by-nc/4.0/

\section{REFERENCES}

1. Defazio $\mathrm{G}$. The epidemiology of primary dystonia: current evidence and perspectives. Eur J Neurol 2010;17(Suppl 1):9-14.

2. Palmer B, Macfarlane $\mathrm{G}$, Afzal $\mathrm{C}$, et al. Acculturation and the prevalence of pain amongst South Asian minority ethnic groups in the UK. Rheumatology (Oxford) 2007;46:1009-14.

3. Albanese A, Bhatia K, Bressman SB, et al. Phenomenology and classification of dystonia: a consensus update. Movement Disord 2013;28:863-73.

4. Albanese A, Barnes MP, Bhatia KP, et al. A systematic review on the diagnosis and treatment of primary (idiopathic) dystonia and dystonia plus syndromes: report of an EFNS/MDS-ES Task Force. Eur J Neurol 2006;13:433-44

5. Torres-Russotto D, Perlmutter JS. Task-specific dystonias: a review. Ann N Y Acad Sci 2008;1142:179-99.

6. Dressler D, Altenmueller E, Bhidayasiri R, et al. Strategies for treatment of dystonia. J Neural Transm (Vienna) 2016;123: 251-8

7. Ben-Shlomo Y, Camfield L, Warner T. What are the determinants of quality of life in people with cervical dystonia? J Neurol Neurosurg Psychiatr 2002;72:608-14.

8. Kuyper DJ, Parra V, Aerts S, et al. Nonmotor manifestations of dystonia: a systematic review. Mov Disord 2011;26:1206-17.

9. Zetterberg L, Lindmark B, Söderlund A, et al. Self-Perceived non-motor aspects of cervical dystonia and their association with disability. J Rehabil Med 2012;44:950-4.

10. Jahanshahi M. Psychosocial factors and depression in torticollis. J Psychosom Res 1991;35:493-507.

11. Stamelou M, Edwards MJ, Hallett $M$, et al. The non-motor syndrome of primary dystonia: clinical and pathophysiological implications. Brain 2012;135:1668-81.

12. Cottraux JA, Juenet $C$, Collet $L$. The treatment of writer's cramp with multimodal behaviour therapy and biofeedback: a study of 15 cases. Br J Psychiatry 1983;142:180-3.

13. Gibson HB. Writer's cramp: a behavioural approach. Behav Res Ther 1972;10:371-80.

14. Jabusch HC, Müller SV, Altenmüller E. Anxiety in musicians with focal dystonia and those with chronic pain. Mov Disord 2004;19:1169-75.

15. Hertenstein E, Tang NK, Bernstein CJ, et al. Sleep in patients with primary dystonia: a systematic review on the state of research and perspectives. Sleep Med Rev 2016;26:95-107.

16. Hewlett S, Ambler N, Almeida C, et al. Self-management of fatigue in rheumatoid arthritis: a randomised controlled trial of group cognitive-behavioural therapy. Ann Rheum Dis 2011;70:1060-7.

17. van Kessel K, Moss-Morris R, Willoughby $\mathrm{E}$, et al. A randomized controlled trial of cognitive behavior therapy for multiple sclerosis fatigue. Psychosom Med 2008;70:205-13.

18. Welschen LM, van Oppen P, Dekker JM, et al. The effectiveness of adding cognitive behavioural therapy aimed at changing lifestyle to managed diabetes care for patients with type 2 diabetes: design of a randomised controlled trial. BMC Public Health 2007;7:74.

19. Surawy C, McManus F, Muse K, et al. Mindfulness-based cognitive therapy (MBCT) for health anxiety (hypochondriasis): rationale, implementation and case illustration. Mindfulness (N Y) 2015;6:382-92. 
20. Bernstein CJ, Ellard DR, Davies G, et al. Behavioural interventions for people living with adult-onset primary dystonia: a systematic review. BMC Neurol 2016;16:40.

21. Cusens B, Duggan GB, Thorne $\mathrm{K}$, et al. Evaluation of the breathworks mindfulness-based pain management programme: effects on well-being and multiple measures of mindfulness. Clin Psychol Psychother 2010;17:63-78.

22. Ong JC, Shapiro SL, Manber R. Combining mindfulness meditation with cognitive-behavior therapy for insomnia: a treatment-development study. Behav Ther 2008;39:171-82.

23. Surawy C, Roberts J, Silver A. The effect of mindfulness training on mood and measures of fatigue, activity, and quality of life in patients with chronic fatigue syndrome on a hospital waiting list: a series of exploratory studies. Behav Cogn Psychother 2005;33:103-9.

24. Craig P, Dieppe P, Macintyre S, et al. Developing and evaluating complex interventions: new guidance 2006. http://www.mrc.ac.uk/ complexinterventionsguidance

25. Leventhal H, Leventhal EA, Contrada RJ. Self-regulation, health, and behavior: a perceptual-cognitive approach. Psychol Health 1998;13:717-33.

26. Ajzen I. The theory of planned behavior. Organ Behav Hum Dec 1991;50:179-211.

27. Armitage CJ, Conner M. Efficacy of the theory of planned behaviour: a meta-analytic review. Br J Soc Psychol 2001;40(Pt 4):471-99.

28. Michie S, van Stralen MM, West R. The behaviour change wheel: a new method for characterising and designing behaviour change interventions. Implement Sci 2011;6:42.

29. Mohebi S, Azadbakht L, Feizi A, et al. Review the key role of self-efficacy in diabetes care. J Educ Health Promot 2013;2:36.
30. Bovend'Eerdt TJ, Botell RE, Wade DT. Writing SMART rehabilitation goals and achieving goal attainment scaling: a practical guide. Clin Rehabil 2009;23:352-61.

31. Jinnah HA, Albanese A. The new classification system for the dystonias: why was it needed and how was it developed? Mov Disord Clin Pract 2014;1:280-4.

32. Comella CL, Leurgans S, Wuu J, et al. Rating scales for dystonia: a multicenter assessment. Mov Disord 2003;18:303-12.

33. Tennant R, Hiller L, Fishwick R, et al. The Warwick-Edinburgh Mental Well-being Scale (WEMWBS): development and UK validation. Health Qual Life Outcomes 2007;5:63.

34. EuroQol Group. EuroQol-a new facility for the measurement of health-related quality of life. Health Policy 1990;16:199-208.

35. Brazier J, Roberts J, Deverill M. The estimation of a preferencebased measure of health from the SF-36. J Health Econ 2002;21:271-92.

36. Zigmond AS, Snaith RP. The hospital anxiety and depression scale. Acta Psychiatr Scand 1983;67:361-70.

37. Klokkerud M, Grotle M, Løchting I, et al. Psychometric properties of the Norwegian version of the patient generated index in patients with rheumatic diseases participating in rehabilitation or self-management programmes. Rheumatology (Oxford) 2013;52:924-32.

38. Løchting I, Grotle M, Storheim K, et al. Individualized quality of life in patients with low back pain: reliability and validity of the Patient Generated Index. J Rehabil Med 2014;46:781-7.

39. Schoffer KL, O'Sullivan JD. Charles Dickens: the man, medicine, and movement disorders. J Clin Neurosci 2006;13:898-901.

40. Rinnerthaler M, Mueller J, Weichbold V, et al. Social stigmatization in patients with cranial and cervical dystonia. Mov Disord 2006;21:1636-40. 